\title{
Analysis on Fragmentation Injury of Glare Stun Grenade
}

\author{
Hongxiang $\mathrm{LuO}^{1}$, Yongzhong $\mathrm{Ma}^{2}$, Xiang $\mathrm{Hu}^{3}$ \\ Engineering University of CAPF, Xi'an ,China \\ 1016428164@qq.com, ${ }^{2} 1150993094 @ q q . c o m,{ }^{3} 499571039 @ q q . c o m$
}

Keywords: Injury fragment, Matlab simulation, Injury effect, LS-DYNA

\begin{abstract}
The safety and reliability design of glare stun grenade is an important research direction of new riot bombs. This paper, with the purpose of studying the injury effect of a certain type of glare stun grenade, starting from the fragmentation injuries, analyzes the radius of injury of this glare stun grenade combined with the Matlab simulation software system, providing a theoretical reference for the practical application of this type of glare stun grenade. The research idea of this paper provides referential experience for injury effect of similar antiriot ammunitions. The simulation model built in this paper can be used not only in this type of glare stun grenade, but also in the study on the injury effect of other similar antiriot ammunitions by changing relevant parameters, thus greatly reducing the experimental cost and time, which has certain practical values and application prospect.
\end{abstract}

\section{Introduction}

Nowadays, with the increasingly serious domestic anti-terrorism situation, the glare stun grenade has become the most widely used non-lethal weapon in the anti-kidnapping activities at home and abroad with its numerous advantages of simple structure, portability and convenience, good effects and so on. However, to reach the technical and tactical requirements of the glare stun grenade, the warhead of this missile must be equipped with adequate doses of glitter. The projectile fragments produced from the explosion are likely to cause irreparable damages to the human body in a certain distance, and there are security risks. Therefore, the study on the fragmentation injury effect of the glare stun grenade is of great significance.

\section{Mechanism of Fragmentation Injury}

After the fragments formed, they will penetrate the human body at a quite high speed, and produce shock to the human tissue to cause harms. The injury effect is related to the penetrating velocity of fragments:

1) Injury effects of low-speed fragments $(340 \mathrm{~m} / \mathrm{s})$ : the shock of fragments forward puts pressure on human tissue, so as to form a wound tract or tear when the maximum stress the tissue can bear is reached. If there are no direct damages to vital organs or vessels, the wound is similar to the injury caused by the handheld kinetics weapons.

2) Injury effects of high-speed fragments $(782 \mathrm{~m} / \mathrm{s})$ : the temporary cavity effect. The high-speed fragments transmit the energy quickly to form the temporary cavity, causing damage to the tissue surrounding the wound track. The more energy transmitted to the tissue from fragments, the larger the temporary cavity, and the degree of trauma caused will be more serious.

The determination of Calculation Methods for Fragments Characteristic Parameters. The characteristic parameters reflecting the fragmentation injury mainly include the quantity of fragments, the initial velocity of fragments, the velocity attenuation coefficient of fragments, the mass distribution and spatial distribution of fragments.

1) The Quantity of Fragments and Mass Distribution

The commonly used formula in China is the Mott formula:

Let's assume that the bomb fragments in two-dimension way, and produces cylindrical thin-walled fragments with uniform thickness, and then the mass and quantity distribution formula of fragments is: 


$$
N\left(m_{f}\right)=\frac{M}{2 \mu} \sqrt{e^{m_{f} / \mu}}
$$

Where, $N\left(m_{f}\right)$ is the quantity of fragments with the mass greater than $m_{f}: m_{f}$ is the mass of fragments; $M$ is the mass of shell cases; and $\mu$ is the Mott fragmentation parameters.

2) Initial Velocity of Fragments

The instantaneous expansion velocity at the time of bomb fragmenting is called the initial velocity of fragments. Then the fragments continue to accelerate under the role of thrust, and the air resistance is equal to the thrust when the fragments reach their maximum speed. Finally, the velocity of fragments gradually reduces with the increase in flying distance until they stop movement. Currently the calculation method for the initial velocity is the Gurney equation:

Only consider the cylinder charge:

$$
D_{t}=\sqrt{2 E} \sqrt{\frac{M / M_{T}}{1+0.5 M / M_{T}}}
$$

Where, $D_{t}$ is the initial velocity of fragments; $M$ is the dose of glitter; $M$ is the mass of shell case; and $\sqrt{2 E}$ is the Gurney constant.

The Gurney specific energy is different for different explosives. Gurney himself obtained the linearly proportional relationship between the Gurney specific energy $\sqrt{2 E}$ and the detonation velocity of explosives $D$, and the equation is $\sqrt{2 E}=520+0.28 D$.

3) The Maximum Initial Kinetic Energy and Specific Kinetic Energy of Fragments

The maximum initial kinetic energy is:

$$
E_{t}=\frac{1}{2} M_{t} D_{t}^{2}
$$

In the formula, $\boldsymbol{M}_{t}$ is the weight of fragments. The maximum specific kinetic energy $e_{t}$ can be calculated according to the maximum initial kinetic energy $E_{t}$ :

$$
e_{t}=\frac{E_{t}}{S_{t}}
$$

In the formula, $S_{t}$ is the mathematical expectation for the encounter area of the target and fragments.

During the flying of fragments, due to the irregularities of their motion, $S_{t}$ is usually treated according to the homogeneous alignment theory, and calculated with the average encounter area of fragments. The encounter area of the target and fragments in this paper is treated by a quarter of the fragments' surface area. The maximum surface area can be deduced from the cartridge mass $M_{T}$, cartridge surface area $S_{T}$ and the maximum fragment mass $M_{t}$. Then we can get:

$$
S_{t}=\frac{1}{4} \frac{S_{T} M_{t}}{M_{T}}
$$

4) Attenuation of Fragment Velocity

Under the function of the detonation products, the fragments obtain initial velocity. During the flying of fragments, due to their large initial velocity and small mass, the influence of gravity can be ignored, so only the impact of the air resistance is considered. The motion equation of fragments can be expressed as:

$$
m_{f} \frac{d V}{d t}=-\frac{1}{2} C_{x} \rho S V^{2}
$$


In the formula, $m_{f}$ is the fragment mass; $C_{x}$ is the coefficient of air resistance; $S$ is the windward area of flying fragments; $\rho$ is the air density at the path fragments flying through; and $V$ is the instantaneous flying speed of fragments.

The fragment projectile is the cylindrical, and the fragments are more in diamond and cylinder after the explosive, so the resistance coefficient uses the 1.1708 .

\section{Statistical Analysis of Fragment Distribution}

The following fragmentation effect pictures at the $0.1 \mathrm{~ms}, 0.2 \mathrm{~ms}, 0.3 \mathrm{~ms}$ and $0.45 \mathrm{~ms}$ are selected from the fragmentation effects of the fragment experiment.
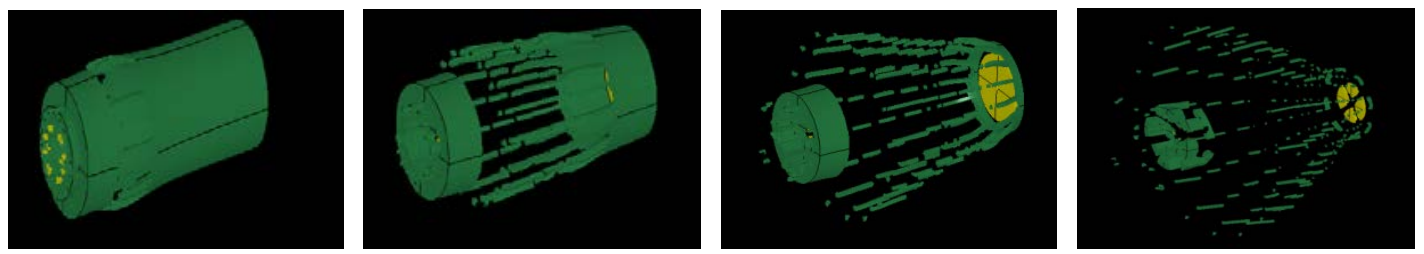

Fig. 1 Fragmentation Simulation Diagram of Projectile at $0.1 \mathrm{~ms}, 0.2 \mathrm{~ms}, 0.3 \mathrm{~ms}$ and $0.45 \mathrm{~ms}$

Through the visibility processing of the results of fragments with the PREPOST, the mass distribution of fragments can be obtained as shown in Fig. 2 :

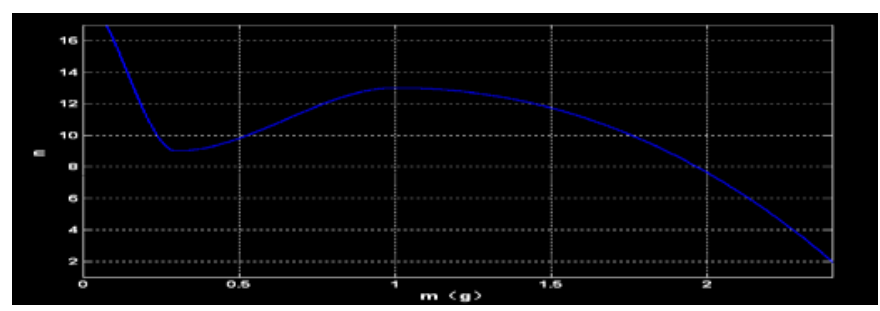

Fig. 2 Mass Distribution Diagram of Fragments

The mass distribution interval of fragments is shown in Table 1

Table 1 Mass and Spatial Distribution Interval of Fragments

\begin{tabular}{|c|c|c|c|}
\hline $\begin{array}{c}\text { Mass Interval of } \\
\text { Fragments (g) }\end{array}$ & Quantity & Percentage (\%) & $\begin{array}{c}\text { Interval of } \\
\text { Scattering Angle } \\
\left({ }^{\circ}\right)\end{array}$ \\
\hline $0.07-0.2$ & 32 & 39.5 & $30-40$ \\
\hline $0.2-0.7$ & 17 & 21 & $50-60$ \\
\hline $0.7-1.7$ & 27 & 33.3 & $0-20$ \\
\hline $1.7-2.4$ & 5 & 6.1 & 0 \\
\hline
\end{tabular}

Analysis and Evaluation of Results. It can be found from the results of simulation that there will be a large number of fragments after the projectile body explodes, among which, larger fragments with mass greater than $1.7 \mathrm{~g}$ account for $6.1 \%$ of the total pieces, and scatter in the direction perpendicular to the main axis of the projectile; smaller fragments with mass less than $0.7 \mathrm{~g}$ account for $60.5 \%$ of the total pieces, and distribute along the direction at the angle of $30^{\circ}-60^{\circ}$ to the main axis of the projectile. The maximum mass of fragments is $M_{\max }=2.40 \mathrm{~g}$, and the minimum mass of fragments is $\boldsymbol{M}_{\min }=0.07 \mathrm{~g}$. The two ends of the projectile produce less fragments, accounting for $24.6 \%$, and the central parts produce more fragments, accounting for $75.4 \%$.

\section{Analysis on Fragments Velocity}

By randomly selecting 11 pieces of fragments 1-K from different mass distribution intervals for the velocity analysis, the time-velocity curve of the fragments can be obtained after the visibility 
processing with the PREPOST:

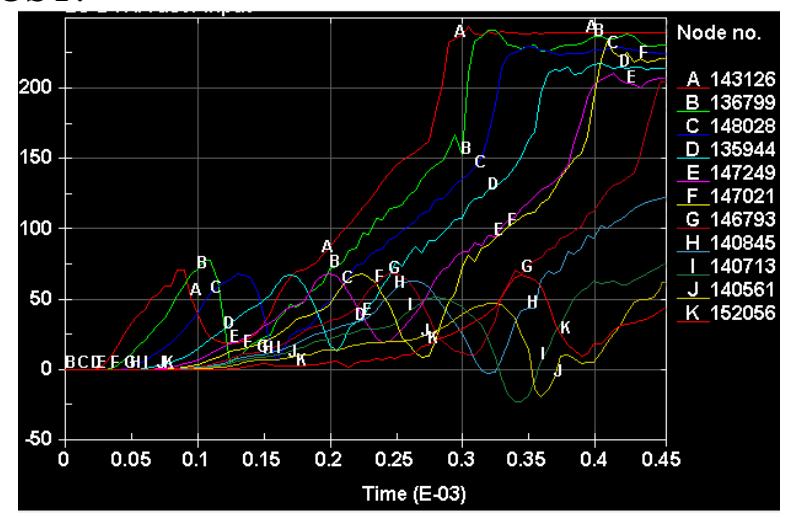

Fig. 3 Fragments Velocity Distribution Diagram

A to $\mathrm{K}$ is the velocity curve of longitudinal eleven fragments. As can be seen from the Fig.3, fragment A can obtain the maximum velocity and its maximum initial velocity is up to $289 \mathrm{~m} / \mathrm{s}$. Fragment $\mathrm{K}$ can obtain the minimum velocity, and its minimum initial velocity is $73 \mathrm{~m} / \mathrm{s}$. it begins to increase at about $0.1 \mathrm{~ms}$, and maximize at $0.3-0.4 \mathrm{~ms}$, and then begins to decline. From front to the back, the maximum velocity decreases slightly but with small falling range.

\section{Assessment on Safety Radius of Fragments}

Selection of Fragments. Because the fragment velocity produced by the glare stun grenade studied in this paper is relatively low, and the injury to the target is mainly caused by the direct hit of low-speed and large-mass fragments, the larger the mass of fragments, the stronger the speed storing ability, and the impact velocity arriving at the target point will be higher, and the kinetic energy will be greater. Accordingly, this paper selects the fragment A with the largest mass as the research object for the following calculation. The fragment A is with the mass of $M_{t}=2.4 \mathrm{~g}$, and the maximum initial velocity of $289 \mathrm{~m} / \mathrm{s}$.

The Minimum Initial Velocity of Fragments Bruising the Skin. The relational expression of the initial velocity and specific kinetic energy of fragments:

$$
v_{\text {min }}=\sqrt{\frac{2 S_{t} e_{\min }}{M_{t}}}
$$

Where, the fragment mass is $M_{t}, e_{\min }$ is the minimum specific kinetic energy of bruising the skin, $v_{\min }$ is the minimum initial velocity of fragments bruising the skin, and $S_{t}$ is the mathematical expectation for the encounter area of the fragments and target.

As the upper part of the cartridge is the firing mechanism with complex structure, we can simplify it and consider it as a projectile with length of $100 \mathrm{~mm}$, and then we can get the following equation after substituting the cartridge mass $M_{T}=41.2 \mathrm{~g}$, the cartridge surface area $S_{T}=113.04 \mathrm{~cm}^{2}$ and the maximum fragment mass $M_{t}=2.4 \mathrm{~g}$ into the formula.

$$
S_{t}=\frac{1}{4} \frac{S_{T} M_{t}}{M_{T}}=\frac{113.04 \times 2.4}{4 \times 41.2}=1.64
$$

Let's assume that this type of glare stun grenade explodes near the sea level, then $H(y)=1$, and the air density $\rho_{0}=1.29 \mathrm{~g} / \mathrm{cm}^{3}$. As larger fragments are not broken and still remain the cylindrical after the explosion of the bomb, the coefficient of air resistance takes the coefficient of the cylindrical fragments $C_{x}=1.17$ and $m_{f}=3.42 \mathrm{~g}$, and the value of the attenuation coefficient $\alpha$ can be obtained after the substitution. 


$$
\alpha=\frac{C_{x} \rho_{0} H(y) S_{t}}{2 m_{f}}=\frac{1.17 \times 1.29 \times 1.64}{2 \times 2.4}=0.51
$$

The following can be obtained after substituting the maximum initial velocity $V_{0}=291 \mathrm{~m} / \mathrm{s}$ and the attenuation coefficient $\alpha$ into the motion equation of fragments:

$$
V=291 \exp (-0.51 x)
$$

The relationship between the specific kinetic energy and distance can be obtained after combining the Eq.7 and the Eq.10:

$$
x=4.06-\frac{\ln e}{1.02}
$$

The safety radius of $1.72 \mathrm{~m}$ can be got after substituting the minimum specific kinetic energy of the fragmentation injury into the above equation.

\section{Conclusions}

This paper carries out a numerical simulation for a series of data including the mass distribution, spatial distribution, initial velocity and so forth of the fragments at the time of the projectile explosion, conducts visibility processing for the results with the PREPOST post-processing tool and Matlab simulation software, and evaluates the injury effect of the fragments to get the distribution rules and injury radius of fragments as well as the injury radius of shock wave. The results are as follows:

1) Injury of fragments to people: it's safe to stay outside the range of $1.72 \mathrm{~m}$; the fragments may bruise or penetrate into the skin with depth of $2-3 \mathrm{~cm}$ between $1.72 \mathrm{~m}$ to $1.12 \mathrm{~m}$; the fragments are lethal within $1.12 \mathrm{~m}$, and may cause serious injury or death.

2) Distribution rules of fragments: there are more fragments with medium and small mass and less fragments with small mass, so no greater harm will be caused to people; the fragments with mass greater than $0.7 \mathrm{~g}$ account for $37 \%$ with the scattering angle between 0 to $20^{\circ}$, indicating that the fragments will mainly harm the part below the legs of people.

\section{Reference}

[1] S.Y. Sui , S.S. Wang. Terminal Effects. Beijing: National Defence Industry Press .2000.(in Chinese)

[2] Y.S. Wang. Criteria Analysis of Warhead Fragment of Zero(Casualty Probability. Journal of Detection and Control,2004,16(2): (in Chinese)

[3] F. Yin, Y.D. Zhang, Q. Fang. Fragment and Its Destroy Effect Produced by Conventional Weapon. Journal of PLA University of Science and Technology: Natural Science Edition, 2005 (1): 50.(in Chinese)

[4] S.Z. Zhang. Explosion and Impact Dynamics. Beijing: The Publishing House of Ordnance Industry,1993.(in Chinese)

[5] Y.S. Huang. Explosive Theories. Nanjing: Nanjing University of Science and Technology Press, 2001.(in Chinese) 